\title{
Managing Policies for Dynamic Spectrum Access
}

\author{
David Lewis, Kevin Feeney, Kevin Foley, Linda Doyle, Tim Forde, \\ Patroklos Argyroudis, John Keeney, and Declan O'Sullivan \\ Knowledge \& Data Engineering Group \& \\ Centre for Telecommunication Value-chain Research, \\ School of Computer Science and Statistics, \\ Trinity College Dublin, \\ Dublin, Ireland \\ Dave.Lewis@cs.tcd.ie, Kevin.Feeney@cs.tcd.ie, \\ Kevin.Foley@cs.tcd.ie, linda.doyle@tcd.ie, timforde@mee.tcd.ie, \\ argp@cs.tcd.ie, John.Keeney@cs.tcd.ie, \\ Declan. Osullivan@cs.tcd.ie
}

\begin{abstract}
The advent of software radio technology and the resulting potential for dynamic access to the radio spectrum presents major new challenges in managing that access. These challenges arise from the likely spread of spectrum access decision-making authority well beyond existing regulatory authorities to a wide variety of co-existing market-based or open-access schemes. Policybased management mechanisms are proposed as a flexible means for defining the rules that determine spectrum allocation dynamically. However, many existing policy based mechanisms rely on a fixed organisation structure and so are insufficiently flexible to support combinations of central allocation, market mechanisms and commons usage. In this paper we present the application of a novel policy-based management mechanism based on self-managing communities to the management of policy authoring authority. We show how an existing implementation could be used to manage a software-based radio system and how this approach provides self-organisation of multiple groupings with differing goals and policies in the allocation of spectrum. This is illustrated by taking real world policy authoring scenario from the world first software radio test license.
\end{abstract}

\section{Introduction}

The advent of software radio offers potential improvements in efficient use of the radio spectrum. By allowing agile, runtime reconfiguration of RF systems, spectral resources can be accessed dynamically, allowing applications to opportunistically exploit portions of the spectrum left unused at any point in time.

Currently the radio spectrum is largely allocated in a command and control manner based on long-range predictions of various licensees, e.g. TV broadcasters, military and emergency service users. This is coupled with the allocation of portions of spectrum to commercial licensees. In recent year some allocation has been on a competitive basis, e.g. through auction of $3 \mathrm{G}$ licenses, however the license terms are still long lived. This has led to under-utilisation of spectrum. It has also, arguably, slowed innovation in technologies for spectrum utilisation, as indicated by the 
acceleration of innovation in unlicensed bands, where utilisation technology is not linked a priori to the opportunity to access the spectrum.

To improve the utilisation of the spectrum, more dynamic access schemes are now being considered by regulators, in particular the F.C.C in the US and the U.K.'s Office of Communication (OfCom). This consideration is supported by advances in the development of agile radio technologies. In particular, there has been a recent trend toward performing more of the functionality in radio transmitters and receivers for data communication in software. Such software-based radio allows radio devices to dynamically switch between different bands, encoding schemes and protocols through software updates or runtime reconfiguration. Such reconfiguration could be made highly dynamic, as suggested for so called cognitive radio, where transmitters sense the spectrum for opportunities to make use of currently unutilised bands [mitola]. Cognitive radio executes a classic autonomic control loop in that it monitors its RF context, analyses the current opportunities then plans and executes a course of action, e.g. to access a particular band with specific power constraints, protocols and encodings. Consistent with this view of cognitive radio as an autonomic communications system, proposals are emerging for the governance of cognitive radio using policy-based management. The U.S DARPA Next Generation Communication (XG) project has proposed a policy-based management framework for cognitive radio [xg-vision]. This allows regulatory rules to be encoded as declarative rules that are executed at runtime as part of the autonomic control loop that implements cognitive radio.

However, central to the idea of dynamic spectrum access is not just that it leads to greater innovation in radio technology, such as cognitive radio, but that such innovation is linked to innovation of the use of the spectrum to meet commercial and social goals. As such, it should not be the aim of regulators to set the rules that are directly enforced in cognitive radio implementation. Instead, regulators will provide a framework of rules within which other bodies will be delegated authority to design the specific rules to which cognitive radio, or other software-defined radio schemes, will adhere. To maximise the opportunity to innovate in the combination of software radio technology and dynamic spectrum access policies a highly disaggregated approach to the delegation of policy-making authority should be encouraged. For example, policy-making authority could be delegated to municipal authorities to best meet local social conditions or different spectrum trading commodity markets could be established in different bands or regions to allow parallel experimentation with market rules. Such an approach may also result in value chains of policy-making authority, with secondary markets being established to satisfy niche trading requirements or regional sub-authorities being established, e.g. an airport being given policy-making authority by the municipal authority in which it is located. This will also allow more responsive tailoring of policies to local conditions. Tailoring can be tuned to geography, the bands subject to the authority, the commercial and social goals guiding policy-making, the density of users/terminals and the range and balance of applications using the spectrum in that locale.

In this paper we identify some shortcomings in the DARPA XG policy framework in its ability to handle a disaggregated ecology of policy making authorities. We propose how an existing scheme for community-based policy management can address these shortfalls and illustrate this with some case studies based on the real 
world policies needed under the world first software radio license that was recently allocated to the Centre for Telecommunication Value-chain Research (CTVR) by the Irish regulator, COMREG.

\section{Policy Management of Software Radio}

The DARPA XG Policy Language Framework aims to provide a means by which machine understandable policies can be defined in a highly flexible and traceable manner [xg-policy]. The design of the language aims to support the frequent changing of policies that apply to a particular radio system. These changes may be used to changes in operator policy, changes in the usage context of spectrum or mobility of the radio system between administrative domains where policies differ. In addition, the language aims to support consistency checking of policies and the easy introduction of new language concepts.

To achieve these goals the XG policy language incorporates many features from modern policy languages. One such innovation is the use of ontology-based semantics to capture machine-processable facts about element of policies. Following their successful application in policy languages such as Rei [kagal] and Kaos [uszok], the XG policy language uses a description logic approach for describing facts as standardised by the World Wide Web Consortium in the Web Ontology Language (OWL) [owl] as part of its Semantic Web initiative. Such facts define concepts for use in policies that can be inherited from multiple existing concepts, simplifying the modelling of spectral resources and the extension of such models over time. Such facts can also be used to define constraints over the concepts used in policies. Such OWL based facts allow existing description logic reasoners to be employed in checking the consistency of policies and inferring new constraints on the application of policies.

XG policies consists of a selector descriptor, which allows for easy filtering of relevant policies, an opportunity descriptor, which defines whether a valid opportunity exists and a usage constraint descriptor which defines constraints on the device and environment under which the opportunity can be exploited, e.g. maximum transmit power in the available band. The selector descriptor contains descriptions of the authority who is establishing and enforcing the policy, the frequency range or frequency group, the region and time over which the policy applies and the capabilities of an device able to enforce the policy.

By default all policy rules satisfying the selector for a device that experiences an opportunity and can conform to the opportunity constraints are applied. However, in common with other policy languages, the XG language allows the definition of metapolicies that specify rules about how other policy rules are enforced. In particular, XG allows policies to be groups, either explicitly or by satisfying a logical expression and it allows precedence of policies to be defined explicitly.

The illustrate the use of this language we specify the policy rules that capture the terms of a recent spectrum usage license granted by to the Centre for Telecommunications Value-chain Research (CTVR), a large multi-institute research effort in the Republic of Ireland (www.ctvr.ie), by the Irish communications regulator, COMREG. This license was granted explicitly for the testing of software 
radio and is believed to be the first such license to be granted worldwide. It is thus a fitting subject for analysing the suitability of the XG policy language. Below we use the CLIPS based notation from [xg-policy] to specify the policy rules that define the terms of this license.

No. Encoded Policy in Shorthand Notation

1 (PolicyRule (id P1)

( selDesc S1)

(deny FALSE)

(oppDesc AnyOpp)

(useDesc U1) )

2

(SelDesc (id S1)

(authDesc COMREG)

(freqDesc F1)

(regnDesc R1)

(timeDesc T1)

(devDesc D1) )

3

4

5

6 (FrequencyRange (id band)

(Usedesc (id U1)

(xgx $"(<=$

MaxTransmitPower

TxParam)" ) )

4 (Power (id TxParam)

(magnitude 1.0) (unit W) )

5 (FrequencyDesc (id F1)

(frequencyRange bandA)

(frequencyRange bandB) )

$(\max 2.0925)$

$(\min 2.0675)$

(unit $\mathrm{GHz}$ ) )

7 (RegionDesc (id R1)

(region

TrinityCollegeDublin)

(region

UniversityofLimerick)

(region

DublincityUniversity)

(region

UniversityCollegeCork) ...)
Remarks

-- Overall rule defining

COMREG license. The terms of the license are not concerned with opportunities.

-- The descriptor defining the authority specifying to policy rule (COMREG). The breakdown of the time and device descriptor omitted for brevity

-- Usage descriptor defines

maximum transmit power

-- Maximum transmit power is $1 \mathrm{~W}$

-- The license specifies two bands (details of band B omitted)

-- A $25 \mathrm{MHz}$ band centred on $2.08 \mathrm{GHz}$

-- Some of the names regions are omitted for brevity. The license simply implies that transmitted must be placed on the premises of these institutes so this does not necessarily match exactly the XG models for a geographical point coordinate or a cylindrical volume.

The above policy represents the extent of the license, and thus of the spectrum allocation rules as specified by COMREG. The license then delegates to the CTVR Executive administrative authority for how this is used. Though the DARPA XG policy language version 1 has some mention of the delegation of authority, allowing to be allocated using the fact keyword PolicyAdministrator, it defers guidance on how the delegation of such authority should be modelled to future version. It is the 
complexity of such delegation in the scenarios described in then previous section that we address in this paper.

Even in the relatively simple case of the CTVR software radio license, an analysis of current working structures in CTVR indicated some of the complications that may arise. For instance it would seem reasonable that the CTVR Executive, on receipt of the license give authority for administering the access to the licensed bands in each location to local network administrators. These local administrators may then define rules which indicate which users can access which sub-bands at which times. However, as a multi-institute centre there often emerges the need to support cross-institute research that may result in spectrum access policy conflicts between the proponent of that research and the local administrators. In a relatively cohesive organisation such as CTVR such conflicts can be resolved by direct communication, however unresolved conflicts can be escalated to the CTVR Executive as the organisational arbiter of access control policies. The Executive may itself possess rules for resolving such conflicts, e.g. simple majority of executive committee members. The capture and execution of this policy resolution process is not easily supported in the DARPA XG policy language currently, largely due to limited mean for expressing policies about the authority to define policies and the organisational modelling this requires. In the next section we examine an generic mechanism based on the idea of hierarchical communities of policy makers that could address this deficiency in DARPA XG. Such flexibility in resolving conflicts in spectrum access policies from different bodies is essential if we wish to allow innovation in mediating access, for instance by using marketbased rules to resolve conflicts.

\section{Community Based Policy Management}

Community based policy Management is a new alternative to modelling a dynamic, adaptive system that moves away from the more traditional form of system modelling; role based modelling [feeney]. The system model, in the case of this paper, is concerned with policy determination for dynamic spectrum access. As indicated above, the DARPA XG policy language provides some basic support for grouping policies, for setting rules about policy execution precedence and offering alternatives between policy groups. However, the DARPA XG model does not support sophisticated meta-policies related to the authoring of policy rules. For this, a more sophisticated model of the policy subjects, or authorities in XG terminology, involved in defining policies is required, where groups of policy authors and the relationship between those groups can be represented as the targets of polices. Languages such as PONDER [damianou] allow meta-policies with policy authorship groups as targets, where these groups are defined in terms of organisation roles [lupu]. However, in the type of fluid organisation represented by CTVR, roles do not provide a sufficiently flexible means for modelling the organisation of authority in a way that allows policy authoring rules to be freely changed. The role-based model sees an organisation as a group of individuals and focuses on their positions and their relationship with the resources available to the organisation [sandhu]. As such there is 
often a mutual dependence between the semantics of different roles that means changes to one role causes changes to others in terms of their policy authoring capability. This stems from the tendency to attempt to use roles to model organisations in their entirety. We propose instead a community-based approach to modelling the targets of policy authoring meta-policies in that this better supports incomplete and fluid models of organisational structure. It is our belief that such flexibility will provide necessary if we are to support innovation in the organisational structures and spectrum access value chains that are described in section 1 .

In the community-based system model, we view an organisation as a set of functional units, termed communities, which are linked to each other by means of a natural hierarchy of authority. The actions of authorisation meta-policy within the system are seen as being exerted on these units, i.e., the functional units are targets of such actions. Once an action is performed on a functional unit, the policy authoring properties of this unit may change or develop. The development of properties in a functional unit is described as "emergent properties". These properties are independent of individual actors in the system, i.e. the system users.

A key concept, in the community based policy model, is the idea of hierarchy. All the functional units in a system are placed within a hierarchy of authority. There are two main conditions that the hierarchy imposes on its members:

1. Membership - on each level of the hierarchy all the members of a particular community are members of the community directly above it in the authority hierarchy.

2. Authority - the authority of a community is superseded by the community directly above it in the hierarchy.

The hierarchy of authority emanates naturally from the idea that communities lower down the tree are members of the communities higher up the tree and therefore the goals of lower communities are to aid the higher communities to fulfil their goals. And so on, until the community at the root of the tree, "the organisation", has all subordinate communities striving towards the ultimate goal of the organisation as a whole. The organisation or root community has ultimate authority over all other communities and resources. The entire community hierarchy tree is itself considered a resource that can be managed with ultimate authority by the root community, and in a limited capacity, by communities lower down the tree. Communities higher up the organisational tree can issue a mandate to communities lower down the tree that delegate authority to a particular sub-community. On receipt of this mandate, the subcommunity (e.g. an executive) can now control the organisation at this level of the tree and below. Mandates are issued by the root communities by means of policies, written by the root community, that allow the selected sub-community to act as an agent of the root community.

Figure 1 shows a sample hierarchy of functional units with in the organisation of the broadband spectrum users in the Irish republic; otherwise known as communities. COMREG has the authority to determine the area of the spectrum the CTVR has authority over. Although CTVR and COMREG are on the same level of the hierarchy tree, one appears to have authority over the other. This may seem to contradict the principle of a hierarchy of authority model. However, policies will have been written by "All Irish Spectrum users" that delegate authority to COMREG to determine the 


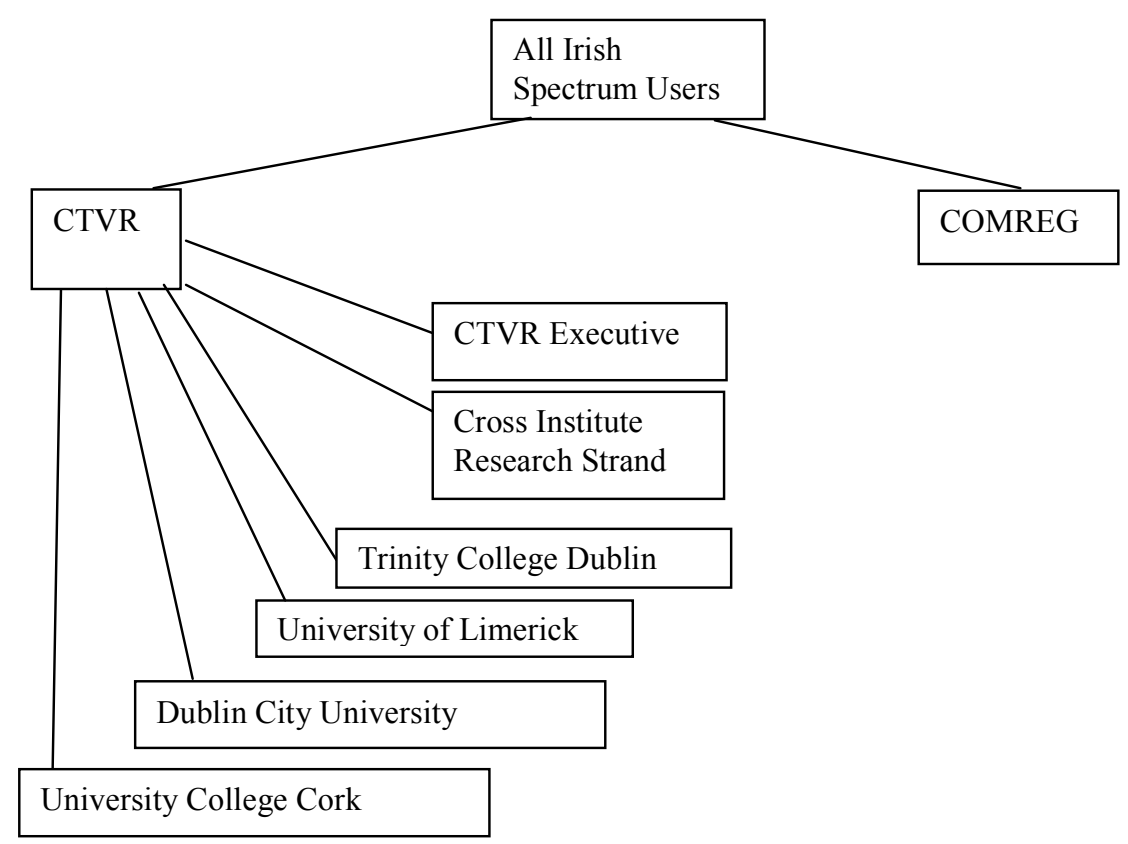

Fig. 1. Community-based policy management scheme for CTVR Software Radio test license

allocation of the spectrum to CTVR. Likewise, CTVR will write a policy to delegate authority of the spectrum allocation to the CTVR executive. In a scenario where the University of Limerick and Trinity College Dublin are running experiments that both requires the full use of the resource, the individual authorities they possess will preclude them from proceeding with the experiment without resolution of the conflict. The conflict is escalated up the tree until the node that has the authority over both competing communities is reached. In the case of this example this is the CTVR executive. The policies of CTVR executive are examined to reveal that the authority to administer full control the full spectrum has been delegated to the executive. The executive then makes a decision, based on its policies, which university should be allowed temporary full control of the resource, to run its experiment. The decision of the executive is passed upward to the CTVR group and then down to the spectrum users. This cycle is know as the "escalate-delegate" request cycle and is the main concept in conflict resolution. By allowing this cycle to be driven by organisational rule, this scheme move us towards the idea of an 'autonomic organisation', i.e. an organisation where conflicts are naturally identified and resolved through to day-today administrative structure.

However, the definition of hierarchical authority is insufficient to describe all forms of existing human organisation. There are many examples of human organisation where the hierarchy of authority of functional units with respect to resources does not hold - more general functional units do not always possess paramount authority over more specific ones. For example, a supply chain is a coordinated system of entities, activities, information and resources involved in 
moving a product or service from supplier to customer. The entities of a supply chain in dynamic spectrum access could consist of different forms of markets for exchanging commoditise portions of the spectrum under different sets of rules appropriate to device and frequency capabilities or application needs, e.g. terminal link or back-haul link in mesh networks. Supply chains are often formed by a collection of autonomous organisations, each with its own hierarchy of functional units and attendant hierarchy of authority. However, in the case of supply chains in dynamic spectrum access, it is frequently the case that the spectrum, as the primary resource in question, is not exclusively controlled by any of these organisations. Thus, it may be the case that none of the participating organisations has authority to take decisions relating to this resource without consulting the other partners according to the agreement that forms the basis of the chain. Therefore, if we want to model the relationship between functional units and resources for the purpose of applying our community model, it is the community that represents the entire supplychain that has authority over this database resource. The individual organisations that constitute the chain are now communities positioned beneath the community that represents the entire supply chain.

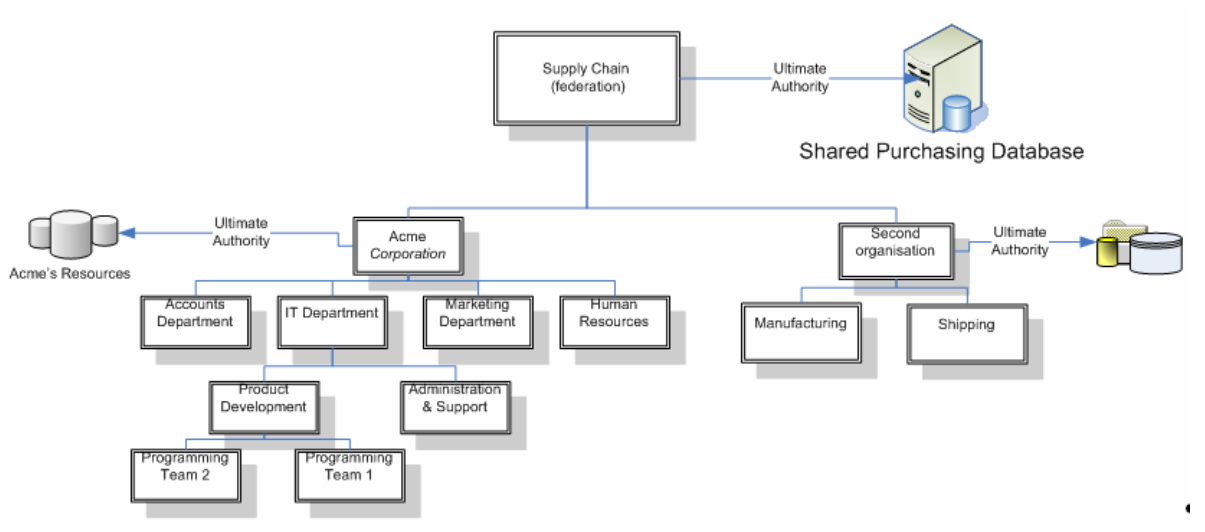

Fig. 2. Simple Schematic of Functional Units' Authority over Resources in Federation

In this case, the hierarchy of authority reflects our community model of the organisation only with respect to those resources which the overall supply chain possesses ultimate authority for. With respect to all other resources, including the resources representing the structures of the constituent organisations, ultimate authority remains with the communities representing the autonomous organisations that make up the supply chain. However, there is little problem in incorporating this type of organisational arrangement into our model. The hierarchy of authority still applies, but the hierarchy of authority for each resource is rooted in the community that possesses ultimate authority for the resource. Thus, the supply chain community does not possess any authority over the non-shared resources of the autonomous organisations that constitute it since ultimate authority for them is possessed by the community representing the organisation. It is only with respect to the shared resources that the top-level community is at the top of the hierarchy of authority. 
Thus the location of the root community in the hierarchy of authority is dependant on the resource being acted upon and is equal to the community which possesses ultimate authority for that resource.

The type of organisational model described above in relation to the supply chain example, we refer to as a federation. It is distinguished from our definition of the organisation above by the fact that the root community possesses ultimate authority for only a subset of the resources managed by the overall community hierarchy. By incorporating the concept that ultimate authority for resources can be distributed among the communities in the hierarchy and by making the community with ultimate authority for a particular resource the root node in our hierarchy of authority for that resource, we can generalise our model to incorporate a very wide range of human organisational forms. The organisation described above - the traditional concept of the organisation - becomes a special case of an organisation, distinguished by the fact that ultimate authority for all resources controlled by the community structure is possessed by the top-level community. So, for example, if we were to consider that the various autonomous organisations involved in our example of the supply chain were to merge and adopt a traditional company form, we would merely need to move ultimate authority for all of the resources to the root community in order to model the change.

Once the community model has been defined, further enrichment of the model is required. Namely: the modelling of the resources available to the community. To model a resource in community based policy management, there is a requirement to separate a particular resource into two distinct sub-units: actions and targets. In the case of the example previous, the broadband spectrum is considered to be the resource. COMREG may wish to apply access control to that particular resource. There must be a target tree defined for the broadband spectrum. The target tree does not necessarily reflect the native hierarchical structure of the resource, but rather the access control requirements of the root community. Thus the tree for a spectrum resource could divide up spectrum by region or frequency or time or other application- or device- dependent capability, or some suitable combination of all of these to form spectral quanta thought suitable for commoditization of the spectrum. Also, as this is a hierarchy model may be possible for the course quanta defined by say a national regulator such as COMREG, to be autonomously subdivided in the finer grained quanta for the purposes of a secondary market.

Leading on from the idea of a target tree, is the action tree. Each resource available to the community has finite number of actions that may be performed on it. The action tree models these finite set of actions in a hierarchy. Therefore an entity that has authority to perform an action on a particular target also possesses the authority to perform any actions beneath the specified action on the action tree on the same target. With regard to the dynamic spectrum allocation model, the "action tree" is basic in structure. However in scenarios such as database administration, the action trees become far more complex and can span several levels.

There is one final part of the community based policy management model that must be implemented to allow for the modelling of real world problems. The relationship of communities, resources and members must be more clearly defined. Thus far we have a model of the organisation in terms of its communities. Further, we have a model of the authority that these functional units possess over the resources 
of the organisation. We have now added a means of mapping the individuals that make up the organisation to these communities as members. However, we can not simply assume that each of the individual members can exercise the authority over the resources possessed by those communities that they are members of. For one thing, recall that in our model the root node of the hierarchical structure of the organisations is the community that represents the entire organisation and that this community possesses absolute authority over all of the resources controlled by the organisation. Further recall that each individual who belongs to the organisation is by definition a member of the root community. If each individual was able to exercise the authority of each community to which he belongs, each individual would possess ultimate authority over all of the organisation's resources through his membership of the root community and the community beneath the root would be obsolete in terms of informing access control decisions. Besides, it is rarely desired that every single member of an organisation should possess absolute authority over all of the resources controlled by the organisation!

Therefore, the final element of our model introduces the concept of a community policy set. Each community possesses a set of policies which dictate how the community's authority over resources can be acted upon by the members of that unit. In terms of our community model of the organisation, which considers the communities to be emergent entities with autonomous agency, we consider the community policy set as the decision making mechanism of the community. Often this would represent some form of collective decision making, e.g. majority voting in a committee style community, or more relevant to dynamic spectrum access, market rules (e.g. auction/bidding rules) for commercially based allocation, or fairnessoriented rules for open commons based access. Thus, when a community policy permits an individual member to exercise the authority of the community to act upon a resource, we say that she acts on behalf of that community and we consider her to be acting as an agent of the community which has approved her actions through its policies.

In summary, the community based policy management model consists of: defined functional units (communities) in a natural hierarchy of authority, a resource authority model that access control is required, a defined authority exercised by these communities on the various resources known as an assigned resource authority, a mapping of individual actors in the system to the communities that defines them as members of communities, and a policy set that defines the authorities possessed by actors in a community that those actors may exercise on behalf of that community. The combination of all these gives a rich dynamic model of an organisation or system that allows for easy distribution of authority as well as a powerful means of conflict resolution. Community based policy management is well suited to the specific problem of dynamic spectrum allocation and is highly given the extremely fluid nature of this problem.

\section{Applying Community Based Policy Management to DARPA XG}

The community based policy management scheme has been implemented as a webbased service using PHP, with a MySQL back end. This offers a comprehensive web 
interface for authoring community-based policies and meta-policies through a unified interface (see figure 4). This interface also reports on the detection of static (or modal) policy conflicts and identifies the point in the community hierarchy from where the conflict arose, and thus at which it must be ultimately resolved.

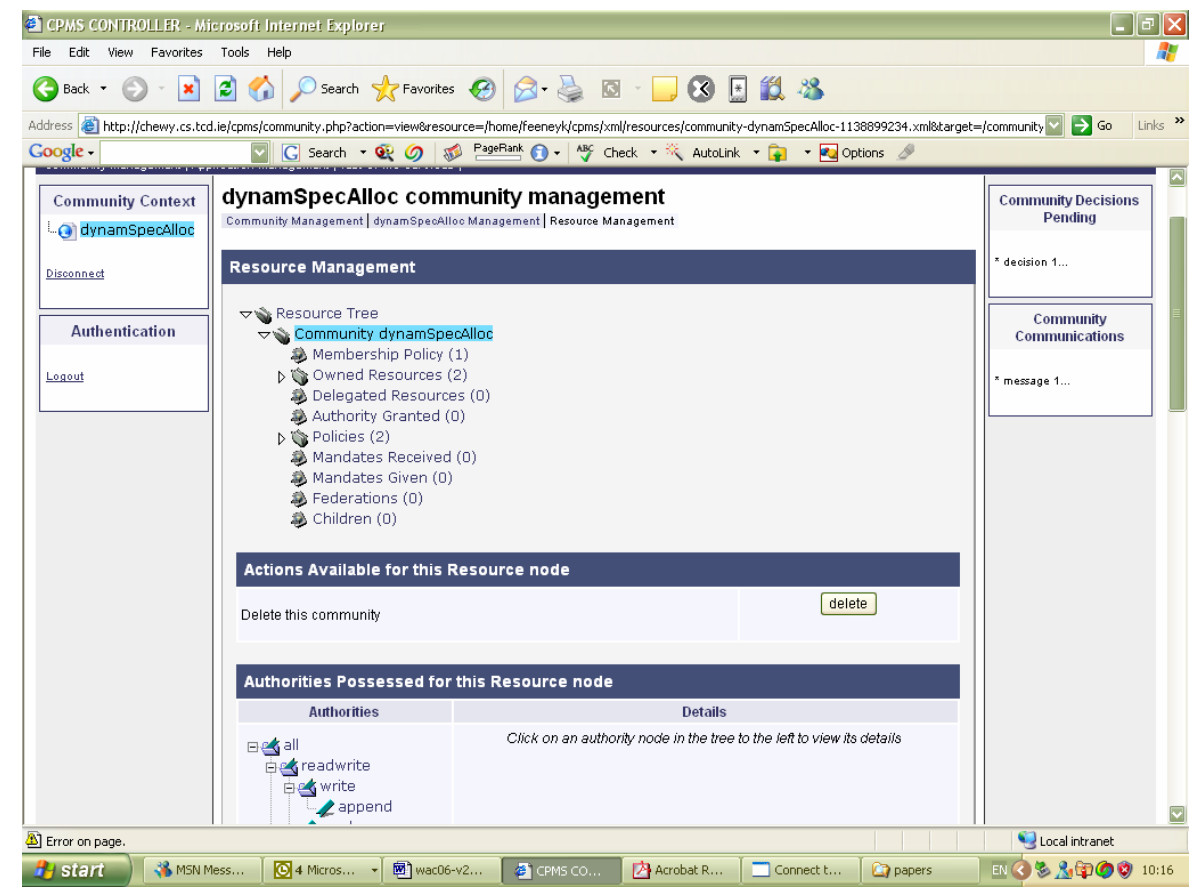

Fig. 3. Sample of the web interface to community

The server also offers the means to offer a policy decision service to other entities. This has been designed to offer a range of easy integration options. To date the policy decision service has been used to integrate the service with a PHP-based collaborative web portal for open source software development [feeney] via a direct PHP call and also to integrate with a decentralised context distribution service via an XML-based Pub-Sub interface. On this basis we are confident that community-based decision making could be readily integrated with implementations of DARPA XG policy decision points as they emerge.

Ultimately, however, our aim is to integrate the community-based policy concepts directly into the DARPA XG policy language using the extension mechanisms it provides. Such extensions should include the following:

- A means of defining a spectrum quanta as a resource. This could be based on existing XG descriptors for frequency, region, time and device descriptors, though anticipate an application descriptor will also be required.

- A means for defining rules for community membership, community mandates and community decision making mechanisms. 


\section{Conclusions and Future Work}

In this paper we identify weaknesses in the DARPA XG policy language that prevent it being used to fully support flexibility and innovation in defining policies for dynamic spectrum access. Using even a simple model of a new software radio license we identify a lack of support in the language for modelling the organisational subject of policies, which in turn restricts the extend to which meta-policies can be used to define means, e.g. markets or fair open access, for sharing resources. We describe an existing policy-based management scheme based on the concept of communities and hierarchical authority delegation between them that can be used to provide such features in DARPA XG. In addition we show how the community based scheme can support the delegation of policy making authority in value chains, which regulatory trends indicate will become an important feature of dynamic spectrum access provision in the future.

Our future work will focus on using our existing community based management service for administration of the CTVR software radio licence. As part of this we aim to integrate community based policy management features as language extensions to DARPA XG.

This integration also throws up a couple of interesting research challenges in policy languages. Firstly, supporting ontology-based extensions to a policy language promises incremental improvement in it expressiveness, however it will be necessary to be able to handle the impact of extension on system with existing rules with elements that are effected by language extension, without the need of bring the system down and re-analysing all existing policies in the context of the extensions. For instance the formation of a new value chain between two dynamic spectrum brokerages may result in resources modelled by one becoming a subclass of a resource used in the other. In this case, all policies related to the latter resource suddenly also apply to the former, raising the possibility of a new set of unexpected policy conflicts. Tools for handling such conflicts without necessarily disabling the policy rules in question would be vital to the smooth operation of frequent value chains formation. A second research issue relates to the development of large policy sets. a key benefit of the community based scheme is that it allow policies to be incrementally introduced as the organisational model grows organically to meet the needs of the organisation or value chain. However, choices in seeding the structure of an organisation can have a strong impact on the efficiency of subsequent organisational change. Poor seeding may result in large numbers of conflicts results form each future change or conflicts that require further resource or action modelling to solve. Such outcomes can be regarded as negative metrics of the policy set so tools to predict the value of such metrics given a set of seed policy structures could provide invaluable. We are currently examining the potential of genetic algorithms and constraint programming techniques as a basis for such tools.

\section{Acknowledgements}

This research was partly supported by Science Foundation Ireland under grant no. 03/CE3/I405, and by the Irish Higher Education Authority under the M-Zones programme. 


\section{References}

[damianou] Damianou, N., Dulay, N., Lupu, E., Sloman, M., (2001) “The Ponder Policy Specification Language” ,Proc. Policy 2001: Workshop on Policies for Distributed Systems and Networks, Bristol, UK, 29-31 Jan. 2001, Springer-Verlag LNCS 1995, pp. 17-28

[feeney04] Feeney, K., Lewis, D., Wade, V. "Policy-based Management for Internet Communities", in proc of 5th IEEE International Workshop on Policies and Distributed Systems and Networks, IEEE, 2004

[kagal] Kagal, L., Finin, T., Joshi, A., “A Policy Language for A Pervasive Computing Environment", IEEE 4th International Workshop on Policies for Distributed Systems and Networks, June 04, 2003

[lupu] Lupu, E.C, Sloman, M. "Conflicts in Policy-Based Distributed Systems Management", IEEE Transactions on software engineering, vol. 25, no. 6, November 1999.

[mitola] J. Mitola III, G.Q. Maguire Jr, "Cognative Radio: Making Software Radios more Personal”, IEEE Personal Communications Magazine, vol 6, pp 13-18, August 1999

[owl] World Wide Web Consortium (W3C), Web Ontology Language (OWL), www.w3.org/2004/OWL/, Visited Mar 2005

[sandhu] Sandhu, R.S. et al., "Role Based Access Control Models", IEEE Computer, vol. 29, no.2, 1996, p 38-47

[uszok] Uszok, A., et al. "KAoS Policy and Domain Services: Toward a Description-Logic Approach to Policy Representation, Deconfliction, and Enforcement" Proceedings of IEEE 4th International Workshop on Policies for distributed Systems and Networks, June 2003 Lake Como, Italy pp 93-99

[xg-policy] DARPA XG Working Group "DARPA XG Policy Language Framework, Request for Comments", version 1.0, prepared by BBN Technologies, Cambridge MA, USA, April 162004

[xg-vision] DAPRA XG Working Group, "The XG Vision Request for Comments". Version 2.0, prepared by BBN Technologies, Cambridge MA, USA, January 2004 